\title{
LOS ABUSOS SEXUALES: EL RIESGO DE SER MUJER
}

FÉLIX LÓPEZ SÁNCHEZ

Universidad de Salamanca

\section{INTRODUCCIÓN}

Los seres humanos podemos amar, pero también odiar. Tenemos manos con las que podemos acariciar, pero también golpear, estrangular y matar. Por eso podemos usar la sexualidad con fines muy distintos. En efecto, la sexualidad humana ha dado un salto cualitativo respecto a la sexualidad animal, que está anclada en el reino de la necesidad -su cerebro y sus hormonas regulan sus deseos y su conducta-. Este salto coloca a la sexualidad humana en el «reino de la libertad»-expresión que hemos acuñado recientemente para indicar la especificidad de la sexualidad humana-: podemos y debemos tomar decisiones sobre nuestra conducta sexual. Las personas no solo regulan su sexualidad desde sus procesos fisiológicos y la parte instintiva de su organismo, sino que pueden y deben tomar decisiones sobre su sexualidad, son libres de masturbarse o no, buscar tener relaciones sexuales o renunciar a ellas, controlar su deseo o darle curso, etc.. Decisiones sobre la conducta de masturbación, aunque en este caso su libertad es máxima, dado que cada persona es propietaria de su cuerpo y de su intimidad; y decisiones, sobre todo, cuando se trata de conductas sexuales que involucran a los demás. En este último caso, las dos personas son propietarias de su cuerpo y de su intimidad, cada cual de sí misma, por lo que la actividad sexual debe ser consentida, querida por ambas. Toda violación de la libertad del otro, llegando a imponer por cualquier medio una determinada actividad sexual, es una grave violación de la libertad sexual, contra la propiedad del cuerpo y de la intimidad, contra la dignidad que las personas podemos y debemos atribuirnos unas a otras.

El hecho de que la sexualidad humana haya alcanzado el REINO DE LA LIBERTAD ${ }^{1}$ hace especialmente valiosa y significativa toda conducta sexual

1. López, Félix: Educación sexual, Madrid, Biblioteca Nueva, 2005. LóPEZ, Félix: Educación sexual de los hijos, Madrid, Pirámide, 2005. 
que involucra a dos personas. Consintiendo pueden disfrutar de la sexualidad, el placer -la actividad sexual está premiada por la naturaleza con el placer-y los afectos interpersonales (ternura, empatía, enamoramiento, amistas, amor, etc.). En este sentido, la sexualidad es una dimensión llena de posibilidades positivas que nos permiten buscarnos, encontrarnos, disfrutarnos, vincularnos $y$, si lo deseamos, tener descendencia.

Pero este hecho, el que podamos tomar decisiones y dar significados a la sexualidad, hace también esta dimensión humana especialmente peligrosa cuando la persona la quiere instrumentalizar, tratando a la otra persona como un objeto, no respetando su voluntad o incluso buscando hacerle daño con la propia actividad sexual. Por eso la sexualidad humana puede dar lugar a lo mejor (consentida, placentera, llena de ternura, empatía y afecto) o a lo peor (hostil, violenta, destructiva). En realidad, como nos decía Sartre, el infierno puede estar en los otros. Hay personas que usan de forma perversa la sexualidad, haciendo daño a los demás, no respetando su voluntad y, en algunos casos, hasta disfrutando con su dolor. Hasta ahí puede llegar la perversión sexual humana.

Los atropellos o delitos contra la libertad sexual, con independencia de la denominación que tengan en cada país, son muchos y pueden ser clasificados desde distintos puntos de vista (especialmente desde el tipo de conducta sexual que implican y desde las estrategias de que se sirve el abusador o agresor):

- Mutilación de partes corporales de claro significado sexual, olvidando que el cuerpo es de cada persona no de la cultura, de un supuesto Dios o de los padres.

- Contacto corporal con significado sexual (buscando excitarse o excitar) sin el consentimiento de la persona afectada.

- Contacto sexual especialmente significativo de forma que se llegue a cometer violación por coito vaginal, anal o actividad sexual oral: Violación.

- Formas de delito sexual aunque no impliquen contacto sexual:

* Comentarios o proposiciones sexuales que persisten a pesar del rechazo o que son vejatorios.

* Exhibicionismo que se hace obligado compartir.

* Voyerismo o violación de la intimidad sexual de otras personas.

- Formas de delito con motivaciones económicas: producción o venta de pornografía infantil, explotación sexual infantil (mal llamada prostitución infantil) $)^{2}$.

- Formas de delitos sexuales por servirse de una relación de poder (laboral, docente, profesional, etc.), para obtener favores sexuales, bajo el supuesto explícito o implícito de sufrir consecuencias negativas o recibir premios si se acepta esa relación: Acoso Sexual.

Sobre todos estos delitos, sin excepción, se pueden asegurar dos cosas fundamentales en relación con el género: las víctimas son con mucha más

2. LÓPEZ, Félix y Guijo, Valeriana: «Explotación comercial e instrumentalización sexual de menores», Intervención Psicosocial, 12 (2003), pp. 65-81. 
probabilidad mujeres (en algunos casos exclusivamente mujeres, como en la mutilación, y otros muchos, casi siempre mujeres) y los agresores son casi siempre hombres. Por todo ello, podemos decir, aunque haremos precisiones más adelante, que la violación de la propiedad del cuerpo y de la intimidad sexual está estrechamente relacionada con el género.

\section{LOS ABUSOS SEXUALES A MENORES}

Dentro de un sin fin de delitos contra la libertad sexual hablamos de abusos sexuales a menores cuando «un adulto, en razón de su asimetría de edad, implica a un menor de edad, antes de la edad de consentimiento de los menores, en actividades sexuales. También puede cometer este delito otro menor, si hay una clara asimetría de edad con la víctima, o si recurre a cualquier forma de coerción» $»^{3}$. Por tanto, para precisar el concepto: se trata de un delito contra la libertad que pueden cometer los adultos, pero también los menores. Los adultos por mera asimetría de edad (cuando los menores no pueden consentir) o porque violentan la voluntad del menor, después de la edad de consentimiento. Los menores, porque también puede haber una clara diferencia de edad con el otro menor (por ejemplo, un chico de 16 años con una niña de 6 años) o porque usan alguna forma de coerción (por ejemplo, amenazar con una navaja a otra persona de su misma edad).

En todo caso, en esta definición, los códigos penales pueden y deberían fijar lo que es minoría de edad (en España, por debajo de los 18 años), cual es la edad de consentimiento (en España, a partir de los 13 años) y cual es la asimetría de edad que hace la actividad sexual delictiva (en España, no se indica). Esto quiere decir que, si un adulto implica en actividades sexuales a una persona menor de 13 años, se trata siempre de un abuso sexual. Si es mayor de trece años sólo hay delito si el menor no consintió, lo que plantea profesionalmente graves dudas, ya que un chico o una chica de trece o catorce años, por ejemplo, puede ser involucrado en actividades sexuales con adultos realmente peligrosas, aunque las consienta, sin que el menor sea muy consciente de ello. Por ello, hay un debate sobre la conveniencia o no de introducir el concepto de "consentimiento informado", es decir, que el menor tenga conocimiento de las implicaciones que su actividad sexual puede tener. Pero se trata de un concepto poco aceptado por numerosos juristas.

Es importante incluir en el concepto de abusos determinadas conductas de otros menores, porque más del $60 \%$ de los que cometen agresiones sexuales comienzan a hacerlo antes de los 16 años y porque, una cosa es que los menores que cometen abusos sexuales tengan un tratamiento penal y social diferente a los adultos, y otra que queden impunes, generando en las víctimas sentimientos de indefensión e impotencia, entre otros. La respuesta social, penal, de la familia y de los profesionales a los abusos y agresiones sexuales que comenten

3. López, Félix: La prevención de los abusos sexuales y la educación sexual, Salamanca, Amaru, 1997 y LÓPEZ, Félix: La inocencia rota, Barcelona, Océano, 2000. 
los menores es esencial para la erradicación o reducción drástica de este problema.

Es evidente que en el concepto de abusos sexuales no se tiene en cuenta el sexo del perpetrador y de la víctima, porque en teoría, cada uno de ellos, puede pertenecer a los dos sexos, aunque, como veremos, el género es fundamental para entender esta problemática.

\section{LAS VÍCTIMAS DE ABUSOS SEXUALES: SER NIÑA IMPLICA EL DOBLE DE RIESGO}

¿Cuántas y quiénes son las víctimas?.

Los estudios científicos internacionales, como es sabido, han confirmado que, al menos por lo que se refiere a occidente, aproximadamente el $20 \%$ de las chicas y el 10\% de los chicos, menores de edad, han sufrido abusos. En otras partes del mundo no se conoce la prevalencia y en otras, por ejemplo algunos países latinoamericanos, la prevalencia es mucho más alta ${ }^{4}$.

El siguiente cuadro refleja algunos de los estudios realizados en diferentes países:

\section{Comparación entre distintos países}

\begin{tabular}{|l|c|c|c|c|c|c|}
\hline \multicolumn{1}{|c|}{ PAÍ́ Y AUTOR } & \multicolumn{2}{c|}{ \% Victimas } & Muestra & Método & Respuesta & Concepto \\
\hline & M & H & & & & \\
\hline Australia, Goldman, 88 & 28 & 9 & 991 (Est.) & Cuest. & $90 \%$ & A \\
\hline Canadá, Badgley, 84 & 18 & 8 & $2008(\mathrm{~N})$ & Cuest. & $94 \%$ & ambos \\
\hline Costa Rica, Krugman, n.p. & 32 & 13 & $497($ Est.) & Cuest. & $99 \%$ & A \\
\hline Dinamarca, & 14 & 7 & $1280(\mathrm{~N})$ & Cuest. & $66 \%$ & ? \\
\hline ESPAÑA, Lopez, 94 & 23 & 15 & $2000(\mathrm{~N})$ & Entrv. & $82 \%$ & A \\
\hline Est. Unidos, Finkelhor, 90 & 27 & 16 & $2626(\mathrm{~N})$ & Telf. & $76 \%$ & A \\
\hline Finlandia, Sariola, 85 & 14 & 7 & $7349(\mathrm{~N})$ & Cuest. & $89 \%$ & A \\
\hline Gran Bret., Baker, 85 & 12 & 8 & $2019(\mathrm{~N})$ & Entrv. & & A \\
\hline Grecia, Agathonos, 90 & 10 & 4 & $746($ Est.) & Cuest. & $95 \%$ & A \\
\hline Holanda, Draijer, 90 & 33 & - & $1054(\mathrm{~N})$ & Entrv. & $49 \%$ & A \\
\hline Irlanda, I.Council, n.p. & 7 & 5 & $500(\mathrm{Lc})$. & Cuest. & & A \\
\hline Noruega, Schei, 90 & 19 & 9 & $1017(\mathrm{~N})$ & Cuest. & $48 \%$ & A \\
\hline Nueva Z., & 13 & - & $314(\mathrm{Lc})$ & Cuest. & $74 \%$ & E \\
\hline Sto Domingo, Ruiz, 87 & -18 & - & $279(\mathrm{Lc})$ & Cuest. & $?$ & ? \\
\hline Suecia, Ronstron, 85 & 9 & 3 & $938(\mathrm{~N})$ & Cuest. & $93 \%$ & A \\
\hline Marcia, C., 2003 & 14 & 26 & $784(\mathrm{Lc})$ & Entrv. & $94 \%$ & A \\
\hline
\end{tabular}

N.P= No Publicado; N: Muestra Nacional; Lc= Muestra Local; Est.= Estudiantes; Cuest.= Cuestionario; Entrv= Entrevista; Telf: Por Teléfono; A: Concepto Amplio; E= Contacto Solo.

4. MALTA, Caesar: «El incesto en Brasil», Tesis doctoral, Departamento de psicología Evolutiva y de la educación, Universidad de Salamanca, 1997. CASTILlo, Marcia: «Los abusos sexuales en Managua», Tesis doctoral, Departamento de Psicología Evolutiva y de la Educación, Universidad de Salamanca, 2003. 
Resultados que, más allá de la variabilidad, ponen de manifiesto, entre otros, los siguientes hechos:

a.- La prevalencia de los abusos es muy elevada en todos los países estudiados. Teniendo en cuenta que estos estudios se consideran, en su conjunto, bastante representativos, quiere decir que estamos ante un problema de grandes dimensiones sociales.

b.- Los abusos sexuales son más frecuentes en las chicas que en los chicos. Aunque en ambos sexos la prevalencia es alta, las chicas duplican el número de casos.

¿Por qué las niñas sufren más abusos que los niños? Una razón parece evidente: los abusadores son casi siempre varones y la mayoría de la población es heterosexual. De forma que, aunque algunos varones heterosexuales abusan también de niños prepúberes, en otros muchos casos los varones heterosexuales mantienen la preferencia sexual hacia las niñas, también con las menores prepúberes. En todo caso, la mayor parte de los niños víctimas de abusos lo son también por parte de varones homosexuales u heterosexuales, no por parte de mujeres.

c.- Las chicas sufren más abusos intrafamiliares, que normalmente son más graves. En todas las investigaciones las chicas sufren más abusos intrafamiliares que los chicos. Por lo que hace relación a España, el 4\% de los abusos sufridos por los chicos fueron por familiar, mientras en el caso de las chicas llegan hasta un $16 \%$.

d.- Los resultados no son, en todo caso, comparables ni generalizables de unos países a otros. En realidad no acabamos de saber, si las diferencias encontradas se deben a alguno de los múltiples factores que hemos analizado más arriba, o son representativas de la realidad de cada país. En este caso, solo los estudios con muestras nacionales podrían tener algún valor.

e.- Las diferentes frecuencias encontradas en cada país no se pueden interpretan de la misma manera. Es decir, al interno de cada una de estas frecuencias se encuentran grandes diferencias.

Por lo que hace relación específicamente a la población española, en relación con la prevalencia, hemos podido confirmar altos porcentajes en diferentes estudios.

En el estudio realizado en España por nuestro equipo ${ }^{6}$ con una muestra representativa de la población española de 2000 sujetos, encontramos que el $18,9 \%$ de los entrevistados dijeron haber sido víctimas de abusos sexuales. En los varones este porcentaje fue del 15,2\% y en las mujeres del 22,5\%.

El que en esta muestra española, el único estudio con carácter nacional, haya más prevalencia que en resto de occidente tiene varias explicaciones. Por un

5. LóPeZ, Félix; CARPINTERO, Eugenio y HeRNÁNDEZ Amparo: «L'inceste: prevalence, auteurs, effet», Revieu Internationale d'education familiale: recherchers e interventions, 6:1 (2004), pp. 23-44.

6. López, Félix; Carpintero, Eugenio; Hernández, Amparo; Martín, María Jesús y Fuertes, Antonio: "Prevalencia de los abusos sexuales en España», Child Abuse and Neglect, 19:9 (1994), pp. 1039-1090. 
lado, nosotros hemos incluido los casos de abusos sexuales ocurridos hasta los 17 años, mientras que bastantes estudios incluyen solo los ocurridos antes de los 16 o incluso los 14 años. Por otro, usamos un concepto amplio, incluyendo los casos de exhibicionismo sufrido de forma individual y sin poder evitarlo, a la vez que, desde el punto de vista metodológico, hicimos una entrevista larga, con más de cien preguntas y en un clima que favorecía la sinceridad.

Si es preocupante que los abusos se den con tanta frecuencia, no lo es menos que se repitan buena parte de ellos. La repetición del abuso aumenta la posibilidad de que los efectos sean especialmente graves, porque la víctima es más probable que se sienta culpable por no evitarlo y porque la relación perpetradorvíctima es más probable que entrañe otros factores de riesgo.

Limitándonos a datos sobre nuestro país, el $55,8 \%$ de los casos de abuso ocurrieron sólo una vez, pero el 44,2\% se repitieron entre 1 y 25 veces más, en nuestro estudio.

Frecuencia de repetición de los abusos

\begin{tabular}{|l|c|c|c|}
\hline \multicolumn{1}{|c|}{ FRECUENCIA } & V & M & Total \\
\hline Nunca & 60,45 & 52,71 & 55,79 \\
\hline De 2 a 3 veces más & 15,67 & 23,15 & 20,18 \\
\hline Entre 4 y 10 veces & 14,18 & 9,36 & 11,28 \\
\hline Entre 11 y 25 veces & 3,73 & 3,94 & 3,86 \\
\hline Más de 25 veces & 5,97 & 9,36 & 8,01 \\
\hline NS/NC & 0 & 1,48 & 0,89 \\
\hline Totales & $\mathbf{1 0 0}$ & $\mathbf{1 0 0}$ & $\mathbf{1 0 0}$ \\
\hline
\end{tabular}

La pubertad es el periodo en el que los niños y niñas son más vulnerables, como se puede ver en resultados de nuestro estudio. Dato éste que confirman otras investigaciones. Aunque es probable que a medida que descendemos en edad algunos casos de abusos no sean reconocidos como tales por los niños muy pequeños o, simplemente, no los recuerden.

\section{Edades de las víctimas}

\begin{tabular}{|l|c|c|c|}
\hline \multicolumn{1}{|c|}{ Edades } & V & M & Total \\
\hline $4-5$ & 2,24 & 4,43 & 3,56 \\
\hline $6-7$ & 6,72 & 12,32 & 10,09 \\
\hline $8-9$ & 14,93 & 16,26 & 15,73 \\
\hline $10-11$ & 11,19 & 16,26 & 14,24 \\
\hline $12-13$ & 26,12 & 24,63 & 25,22 \\
\hline $14-15$ & 28,36 & 16,26 & 21,07 \\
\hline 16 & 10,45 & 9,85 & 10,09 \\
\hline Totales & $\mathbf{1 0 0}$ & $\mathbf{1 0 0}$ & $\mathbf{1 0 0}$ \\
\hline Medias & $\mathbf{1 2}$ & $\mathbf{1 1 , 1}$ & $\mathbf{1 1 , 4 4}$ \\
\hline
\end{tabular}


Por tanto, en los 11 primeros años de vida se producen en nuestro país en torno al $44 \%$ del total de abusos sexuales, cuando aún son prepúberes las víctimas. Eso quiere decir que un numero muy importante de nuestros PREPUBERES, nuestros niños y nuestras niñas, VEN VULNERADOS SUS DERECHOS a la propiedad de su cuerpo y a la intimidad.

Por lo que hace relación a los efectos de los abusos, tanto a corto como a largo plazo, han sido también muy estudiados ${ }^{7}$ :

- Los efectos a corto plazo afectan a la mayoría de las víctimas (en torno al 70\%, en numerosos estudios): reacción de desconfianza, miedo, vergüenza, asco, hostilidad, culpa, etc. En estos efectos no hay duda de que podemos establecer una relación causa efecto.

- Los efectos a largo plazo son más difíciles de estudiar, siendo especialmente complicado establecer relaciones de causa efecto, porque hay otros muchos factores que acompañan al abuso, como por ejemplo que haya pasado un tiempo muy grande entre el suceso y la medida (casi siempre retrospectiva), etc. Por eso es más correcto hablar de asociaciones entre el hecho de haber sufrido abuso en la infancia y tener, por ejemplo, depresión, ansiedad, baja autoestima, etc. en la vida adulta. Estas correlaciones están lejos de permitir establecer relaciones de causa efecto, que naturalmente tampoco se descartan en algunos casos. El hecho de que no haya necesariamente una sintomatología específica y el que influyan muchos factores (el tipo de abuso, su frecuencia, la edad de la víctima, la relación con el agresor, la reacción del entorno, etc.) hace que las generalizaciones sean muy arriesgadas. En todo caso, las personas que sufren efecto a largo plazo no parecen superar el 15 ó 30\%, con todo el relativismo que debe darse a esta información.

No es posible establecer claras diferencias en los efectos en los chicos y las chicas, porque, por un lado, las chicas sufren más abusos intrafamiliares, que suelen ser más graves, mientras, por otro, suele haber una construcción social que hace más vergonzoso el hecho de haber sufrido abusos en el caso de los chicos.

Otra diferencia de género bien establecida es que aunque ambos sexos lo cuentan preferentemente a un amigo o amiga (que les guarda lamentablemente este mal secreto) las niñas se lo cuentan con más frecuencia a las madres, sin duda porque, como en otros temas referidos a la sexualidad, hablan más con ellas y esperan, seguramente, una clara solidaridad de género. Lamentablemente tampoco la mayor parte de las madres reaccionan adecuadamente, tendiendo a guardarle el secreto a la hija y a no denunciarlo.

7. LÓPEZ, Félix: La prevención de los abusos..., op.cit. y LÓPEZ, Félix: La inocencia rota..., op.cit. 


\section{LOS ABUSADORES SEXUALES: UNA CUESTIÓN DE GÉNERO}

Aunque hay algunos casos de abusos sexuales cometidos por mujeres, éstos son muy poco frecuentes y prácticamente nunca se trata de la madre biológica o adoptiva. Los abusos sexuales a menores, a pesar de las excepciones que confirman la regla, son cometidos casi siempre por varones, adultos o menores de edad. Esto es así también en el resto de casos de delitos contra la libertad sexual.

Además de este hecho, indudable, hay otro de gran interés: las niñas que son víctimas de abusos sexuales no tienen prácticamente ninguna posibilidad de convertirse en abusadoras, mientras los niños que han sufrido abusos tienen más probabilidad de convertirse en perpetradores de abusos y otros delitos sexuales que el resto de los varones. Dicho de otra manera: es mucho más probable que esta conducta sea aprendida y repetida por los varones que por las mujeres.

Todo ello nos obliga a pensar una y otra vez cuáles son las raíces (los factores) de género que fomentan, potencian, promocionan el que estas conductas, consideradas en nuestra sociedad como muy graves, vergonzosas e inaceptables, sean aprendidas y repetidas por los varones, aunque no necesariamente, entiéndase bien, y no por las mujeres.

Es este sentido, es importante precisar una falsa creencia muy extendida: si se han sufrido abusos cuando se es menor, es más probable que se acabe cometiéndolos cuando se es adulto. Dicho así es completamente falso, porque no ocurre esto en el caso de las niñas y las mujeres. Por cierto, también un número importante de hombres aprenden a salirse de esta rueda perversa, por lo que no debe hacerse esta profecía fatídica sobre cada niño que ha sufrido abusos. Aún así, es indudable que el ser varón hace más probable este aprendizaje y esta conducta.

Para entender la importancia del género como variable explicativa de los abusos y agresiones sexuales a los menores (también a los adultos) es importante señalar que el género influye de forma transversal a diferentes situaciones, saltando todas las supuestas barreras o factores situacionales':

- Los varones, en general son casi siempre los agresores.

- Lo son más los varones menores, adultos y viejos, que las mujeres de las diferentes edades. El hecho es transversal a la edad.

- Son también mucho más probablemente abusadores los religiosos que pertenecen al clero que las religiosas. Este dato es especialmente significativo en nuestra muestra, porque los varones adultos, pertenecientes a una u otra clase de clero, han llegado a cometer frecuentes abusos sexuales (el $9 \%$ de los abusos sufridos por los chicos), en los internados u en otros tipos de colegios o

8. Los conceptos de sexo y género han sido y son muy discutidos. Entendemos por sexo el dimorfismo biológico hombre-mujer y por género el conjunto de asignaciones sociales y psicológicas que una determinada cultura considera propias de los hombres y de las mujeres.

9. LÓPEZ, Félix: La prevención de los abusos..., op.cit. y LÓPEZ, Félix: La inocencia rota..., op.cit. 
situaciones en que tienen contacto con los menores. Las religiosas, en situaciones semejantes, no han llegado, ni de lejos, a esta cifra (menos del 1\%). Luego el género es transversal al estado religioso o laico. Lo mismo podríamos decir del estado civil.

- Los homosexuales varones (aunque en nuestra investigación no tenemos datos de la orientación sexual de los abusadores) cometen abusos sexuales de forma similar a los varones heterosexuales, mientras que es muy improbable que los cometan las lesbianas. Los abusos y agresiones son transversales a la orientación y al deseo.

- Los varones son los responsables de la casi totalidad de los casos de abusos sexuales a las niñas y de la mayoría de los casos de abusos sexuales a los niños.

De forma que el género, tiene una influencia tan grande que es transversal a factores tan importantes como la edad, el estado civil, el estado religioso y la orientación del deseo del perpetrador y del sexo de las víctimas.

\section{5. ¿POR QUÉ CASI TODOS LOS ABUSADORES Y AGRESORES SEXUALES SON VARONES?}

El que las niñas tengan aproximadamente el doble de posibilidades de sufrir abusos sexuales tiene una aparente explicación clara, ya indicada: casi todos los abusadores son varones y hay más heterosexuales que homosexuales. Por ello, aunque es esperable que los varones homosexuales se interesen más por los niños y aunque hay varones heterosexuales que abusan también de niños prepúberes -más excepcionalmente de adolescentes varones- finalmente se impone el hecho indudable de que los abusadores son casi siempre varones y el hecho menos firme, pero seguro, de que los varones heterosexuales se interesan más por las niñas. Como es sabido en torno al 95\% de la población de varones son heterosexuales.

Pero ¿por qué los varones es mucho más probable que cometan abusos que las mujeres, a pesar de que las niñas sufren más abusos? La respuesta no es clara $y$ es importante reconocerlo.

No tenemos ninguna duda de que hay diferencias en la construcción (proceso de socialización) del género que explican hechos tan relevantes antes señalados. Estas diferencias son conocidas y tienen un alto poder explicativo. Que éstas sean la única y exclusiva explicación de estos hechos, no debe darse por cierto. Es decir, todos sabemos que el proceso de socialización masculino, al menos en numerosas sociedades, tiene contenidos y procesos que favorecen el que los varones recurran a la fuerza, a la coerción, a la presión, al engaño, al soborno, al abuso de confianza y a mil estrategias más para doblegar la voluntad de la mujer adulta o para abusar de un menor, especialmente de las niñas. Pero alertamos de inferencias injustificadas que, sin más, afirman, como una frase ritual, "la explicación es cultural, sólo y exclusivamente cultural».

Vaya por delante que ninguna posible explicación, sea cultural o más compleja, exculpa esta conducta. Por eso insistimos tanto, en los últimos años de nuestro estudio y reflexión sobre la sexualidad, que la sexualidad, en la especie 
humana, ha saltado del reino de la necesidad al "REINO DE LA LIBERTAD», por lo que inexorablemente tenemos toda la responsabilidad sobre nuestra conducta sexual. Ninguna explicación exculpa: todos, hombres y mujeres, tenemos el derecho a que sea respetada nuestra libertad sexual y el deber de respetar la de los demás. Hemos superado el reino de la necesidad, podemos decir NO a una relación sexual y decir Sí a otras relaciones sexuales, ninguna razón biológica o social debe imponernos y llevarnos a imponer ninguna conducta. Esta libertad tampoco se pierde en las parejas o matrimonios convencionales.

\subsection{La masculinidad como causa}

Dicho esto, para no llevarnos a malentendidos, es indudable que la construcción social de la masculinidad, en nuestra cultura y en otras muchas, favorece el que numerosos varones se sientan más inclinados y justifiquen más sus conductas de abuso o agresión sexual. No podemos, tampoco seríamos capaces de conseguirlo, señalar todos esos factores de origen cultural asociados a la masculinidad, pero, a modo de ejemplo, sí indicar algunos de los más importantes (en el contexto general de una sociedad que es claramente sexista y discriminatoria con la mujer, sobre todo, en toda relación en la que pueda verse involucrado directa o indirectamente el poder, la sexual es una de ellas):

- La infravaloración de la mujer y, como caso extremo, la consideración de ésta como un objeto que puede instrumentalizarse a gusto y capricho del varón.

- Atribuirse (los varones) una supuesta necesidad sexual que necesariamente tiene que desahogarse en conductas sexuales a las cuales las mujeres no pueden o no deben decir NO.

- Considerar (en relación con los varones) que no es posible controlar los impulsos sexuales, especialmente una vez que se ha iniciado la excitación sexual.

- Hacer de la seducción una conquista que necesariamente tiene que acabar doblegando la voluntad de la mujer.

- Considerar que el valor de un varón que conquista a una mujer (y más aún a muchas) vale más que quien respeta el NO. El varón aprende a hacer depender su autoestima y valía como varón de su capacidad para seducir, conquistar e incluso doblegar.

- Atribuir mayor valor a los varones que tiene relaciones sexuales con más mujeres diferentes.

- Considerar que las mujeres deben ser, como se dice en argot masculino "castigadas», dominadas, doblegadas, sometidas, etc.

- Atribuir a las mujeres el deseo de ser presionadas, el deseo de decir Sí, cuando dicen No, el gusto por ser obligas o tratadas con cierta violencia. Se puede llegar, a partir del supuesto de que las mujeres no son sinceras, a la creencia de que no hay que aceptar su No.

- El sentido de propiedad de las esposas o, incluso, de las hijas, por lo que no pueden decir No al esposo o al padre.

- El aprendizaje de estrategias de dominación, engaño y violencia como forma de obtener favores sexuales. Estas estrategias las aprenden más los varones que las mujeres, en casi todos los dominios de la vida. 
Todo ello tiene siempre, como correlato, un tipo de socialización de la mujer que es paralela y dependiente de los intereses de los varones: ser condescendiente, pasiva en los inicios de la relación, dulces, cariñosas, y comprensivas con los varones. Había un rito en parte convenido: «tu te resistirás y yo te conquistaré» (pensaba el varón); "tu insistirás, me presionarás, no cejarás hasta conseguirlo y yo, después de una larga resistencia, cederé, aunque solo sea por darte gusto, para que no me abandones...» (podía llegar a pensar la mujer o atribuírsele que pensaba). Bien significativos son algunos hechos: la frecuencia con que las mujeres dicen que la primera vez lo «hice por él» y, lo que es mucho más grave, el hecho de que la mayor parte de las violaciones sean cometidas por un conocido que es, a la vez, amigo o pareja.

Así podríamos seguir el rastro de numerosos factores culturales, bien estudiados en numerosos trabajos de investigación. Los varones, en definitiva son socializados de manera que se les enseña a dominar, a ser más activos, a imponer su voluntad, a presionar y a pelear si fuera necesario, a saberse valiosos si demuestran poder, etc. ${ }^{10}$.

\subsection{La filogénesis como causa}

Pero no debemos dar por cierta y, sobre todo, por exclusiva, esta explicación cultural tan clara, pero, a la vez, tan simple.

De hecho es frecuente encontrar dos simplificaciones explicativas: es "la cultura", como hemos señalado, y es "el deseo de dominación y poder de los varones, que, de hecho recurren más a la violencia». Sin negar el peso de estas razones, creemos que hay influencias más complejas que asientan sus raíces en la filogénesis de los hombres y las mujeres, es decir, en la evolución de la especie.

Para empezar, es fácil demostrar que las dos razones anteriores tienen sus debilidades. Por ejemplo, en relación con la explicación cultural, ¡cómo explicar que en casi todas las culturas los hombres sean los que imponen las reglas del juego a las mujeres?; y en relación con la supuesta explicación de la tendencia a la dominación y el uso del poder y la violencia, ¿cómo explicar que la mayor parte de los abusos sexuales se cometan sin recurrir a la violencia, basándose en estrategias como la confianza, la sorpresa, el engaño, el soborno, etc.?, ¿cómo explicar aquellos casos, frecuentes, en que los hombres que abusan sexualmente de los menores no sólo no recurren a la violencia, sino que se sienten mal si la emplean?

Creemos, con los evolucionistas, que por razones filogenéticas y, más en concreto, el rol que cada sexo desempeña en la reproducción y los aprendizajes hechos a partir de este rol, que hasta hoy (tal vez, como veremos, esto no sea cierto mañana) los hombres están más dispuestos al sexo ocasional que las mu-

10. FINKELHOR, David: Source book on child sexual abuse, Newbury Park CA, Sage Publications, 1986. FINKELHOR, David: "The international epidemiology of child sexual abuse», Child Abuse and Neglect, 18:5 (1994), pp. 4009-4017. 
jeres, que son menos selectivos y que se autocontrolan menos ante una oportunidad de tener relaciones sexuales. La explicación evolucionista era evidente en el pasado, a lo largo del cual hemos hecho aprendizajes ancestrales los hombres y las mujeres: los hombres tienen capacidad reproductora casi ilimitada y su conducta coital tiene sobre ellos menos consecuencias biológicas -embarazo, parto y lactancia- (a parte, claro está de las leyes y convenciones sociales o su sentido de la responsabilidad), por lo que es más probable que busquen tener más relaciones con más parejas; las mujeres tienen capacidad reproductora limitada y, sobre todo, el coito implicaba para ellas una gran inversión y responsabilidad, porque podían quedar embarazadas, tener que parir y amamantar, por ello debían pensárselo dos veces y ser más selectivas con los varones. Estos hechos, según los evolucionistas, mantienen hoy cierta vigencia, especialmente en forma de aprendizajes ancestrales que se transmiten de generación en generación, aunque la están perdiendo, dado que las mujeres han aprendido a evitar el embarazo y los hombres están sometidos a leyes y valores sociales que les hacen responsables de sus hijos biológicos. Por ello, es muy probable que estas razones estén perdiendo peso y que acaben desapareciendo en el futuro, pero aún hoy es evidente que los hombres están más disponibles para el sexo ocasional, sin compromiso ${ }^{11}$. Esta diferencia en la forma de afrontar el sexo ocasional se reproduce en la conducta de masturbación, en todas las edades, de forma que los niños, los chicos y los varones adultos se masturban más que las mujeres de cada uno de estos grupos de edad.

Lo sorprendente es que también hemos encontrado estas diferencias entre los niños y las niñas prepúberes: aunque ambos sexos tienen la fisiología de la excitación y el placer sexual activado, los niños la descubren con mayor frecuencia, están más motivados por la búsqueda clara y directa del placer sexual, de hecho se masturban más y se involucran en más juegos sexuales en los que se excitan y buscan abiertamente el placer. Algunos autores, por cierto, ven como causa de estas diferencias no tanto en la fisiología sexual, sino en la anatomía, dado que la excitación del pene y el placer de esta excitación sería más fácil de descubrir, por ser más grande, estar más externo y más expuesto al contacto que el clítoris de las niñas.

Estas diferencias sexuales entre los sexos es seguro que, a su vez, están influidas por la cultura sexista y más sexofóbica hacia las manifestaciones sexuales de las niñas y las mujeres adultas (de hecho las niñas prepúberes se sienten más culpables, a pesar de tener menos conductas sexuales explícitas ${ }^{12}$ ).

Pero es muy posible que ambas influencias (la filogenética y la socialización sexista ontogenética), sean dos factores que se retroalimentan: diferencias fisio-

11. LOPEZ, Félix: «Diferencias en la conducta sexual entre hombres y mujeres», en Esther Barberá (coord.): Diferencias de género, Perrazos, Madrid, 2004. CARBALHEIRA, Ana: "Relaciones interpersonales y sexuales a través de Internet», Tesis doctoral, Departamento de Psicología Evolutiva y de la Educación, Universidad de Salamanca, 2005.

12. LóPEZ, Félix; GuIJO, Valeriana y Del CAMPO, Amaya: «Prepuberal sexuality», en European Journal of medical Sexology, 44 (2003), pp. 49-64. 
lógicas favorecen diferencias culturales, las sociedades «machistas» hacen más evidentes, exageran y hasta producen una grave discriminación sexista, a partir de aquellas.

Algo parecido debió ocurrir con el hecho de que los roles en la reproducción obligaron a la mujer a estar más vinculada a los embarazos y a la crianza de los hijos, mientras los hombres era más frecuente que se responsabilizaran de la caza y la defensa o las conquistas guerreras: estas actividades pudieron llevar a que los hombres desarrollaran actitudes y conductas más agresivas, mayor fuerza física, se hicieran dueños de las armas y el poder y llegaran a usar estos medios de forma discriminatoria con las mujeres.

Es muy posible, que la interacción y retroalimentación entre los roles sexuales en la reproducción y el hecho de que acabaran convirtiéndose en construcciones sociales más amplias, discriminatorias y claramente sexistas, sean los responsables de la situación del hombre y la mujer hasta nuestros días, aunque hoy carezcan de toda base y sentido.

Aunque algunas personas se puedan molestar, y aunque como hemos dicho hoy los seres humanos estamos muy alejados del reino de la necesidad y el dominio de los instintos, como ocurre en otras especies, hay un cierto paralelismo entre las diferencias hombre mujer y las que se dan entre numerosos mamíferos: en ellos los machos están dispuestos a copular siempre que pueden y con cualquier hembra disponible, mientras éstas, que finalmente son las que se hacen cargo de la cría en solitario o con un rol de maternidad muy especial dentro de la manada, tienen regulada su disponibilidad a través de el "estro» o «celo». ¿Es esta situación la que se dio durante miles de años entre nuestros ancestros prehumanos?, ¿qué y cómo se ha mantenido algo de esta diferencia originaria? Es evidente que los hombres y las mujeres estamos muy alejados de esta situación, pero puede servir de metáfora o ejemplo escandaloso, claro está, de algunas diferencias que, sin duda pueden ser también, en su origen filogenéticas, debidas a los abatares de la evolución de la especie en tiempos en que las relaciones eran, aún mucho más que hoy, relaciones de poder físico y militar, además de relaciones de parentesco y clan.

Si esto fuera así, como creen los evolucionistas, habría que hacer, sin embargo, dos precisiones fundamentales:

* Las diferencias entre los sexos en la conducta sexual son hoy menores que las semejanzas y siempre se encuentran grupos de mujeres o de hombres que siguen un patrón sexual por encima de la media de lo que cabría esperar por su sexo (por ejemplo, un número relativamente importante de niñas prepúberes se masturban más que la media de los niños y un número importante de mujeres adultas aceptan bien el sexo ocasional, incluso mejor que un número relativo de hombres). Ello quiere decir que las diferencias entre los sexos no son rígidas, que los seres humanos tenemos poco preprogramada nuestra vida sexual, que podemos tomar decisiones sobre ella y, por ello, éstas están sometidas a diferencias personales importantes, que en muchos casos superan las influencias del sexo y del género. 
* Si algunas de estas diferencias debidas a distinto rol en la reproducción tuvieron, como parece indudable, influencia original en la construcción de los roles de género, esto no significa que:

a.- Se justifique esta evolución sexista y discriminatoria a lo largo de la historia. Dichas diferencias deben ser denunciadas y superadas. Son, en definitiva, uno de los aspectos más dolorosos y estables de nuestra existencia como especie humana.

b.- Hoy tengan justificación o base alguna, dado que, por un lado, la mujer puede decidir sobre su fecundación y su actividad sexual con la misma libertad que el varón y, por otro, la cultura (en este caso con una influencia en sentido positivo) nos ha hecho aprender a socializarnos de forma que debemos ejercer la paternidad con la misma responsabilidad que las mujeres ejercen la maternidad.

Por ello, con independencia de los análisis históricos que hagamos, en los que no deberemos nunca negar que llegaron a construirse roles de género sexistas y peligrosos para la mujer (por cierto, también para el hombre, aunque un sentido distinto), lo más importante es que tengamos en cuenta que no hay base alguna actual para sustentar ningún timo de discriminación entre la vida sexual del hombre y de la mujer y, por lo que al tema nos ocupa, que no hay ninguna justificación para que los hombres abusen sexualmente de los menores, acosen o violen a las mujeres. Hombres y mujeres, como hemos dejado varias veces claro, podemos y debemos tomar decisiones sobre nuestra sexualidad. Y estas decisiones cuando implican a otros tiene que ser éticas, porque afectan a la libertad de ambos.

\section{LAS EXPLICACIONES INDIVIDUALES}

Aunque, como hemos visto, es indudable que el convertirse en abusador y agresor sexual es una cuestión asociada indudablemente al género masculino, esta asociación no es una vinculación o determinación que haga imposible que haya mujeres abusadoras y agresoras o que conlleve que los hombres sean necesariamente abusadores y agresores. De hecho, la mayor parte de los hombres, no se ven involucrados a lo largo de su vida en ningún abuso o agresión sexual. Ello demuestra dos cosas: que efectivamente la sexualidad humana pertenece al REINO DE LA LIBERTAD (es posible tomar decisiones sobre ella) y que, a pesar de las deficiencias en la socialización masculina, la mayoría de los hombres no sobrepasan estos límites, ubicando su vida sexual en el REINO DE LA ÉTICA.

Por tanto, sin justificación para cometer cualquier tipo de abuso o agresión sexual, ¿qué puede llevar a hombres concretos -también a algunas mujeres, muy excepcionalmente- a cometer semejantes conductas?

La respuesta en este caso es compleja, porque no pueden encontrarse tipos de agresores, sino un sin fin de razones que, en interacción o por sí solas, desencadenan estas conductas. Es decir, los abusos sexuales tienen un origen multicausal y no fácilmente predecible desde el punto de vista social. Entre estas razones cabe destacar las siguientes: 
a.- La falta de autocontrol o impulsividad situacional o más generalizada. Causada por el consumo de drogas, incluido el alcohol, o por características de la personalidad. Hay personas que no controlan, cuando se drogan o por déficit de personalidad, su deseo sexual y que llegan a buscar abiertamente las conductas sexuales, también con menores que no consienten o no tienen edad para consentir.

b.- La falta de una socialización ética adecuada, de forma que hay personas que consideran que pueden usarse los menores para satisfacer sus necesidades sexuales y que consideran legítimas las estrategias para doblegar su voluntad.

c.- Limitaciones importantes en habilidades sociales, de forma que hay personas que no saben o no se esfuerzan en seducir a los iguales, resultándoles menos exigentes o vergonzosas estas actividades con los menores.

d.- Hay personas que disfrutan doblegando la voluntad, sometiendo, vejando, etc., a otras personas, también a los menores, instrumentalizando la sexualidad con otros fines perversos relacionados con el gusto por ejercer el poder o provocar daño.

e.- Hay personas que por razones de aislamiento social o convencionales (el clero, por ejemplo) o institucionales (estar en prisión o en un internado) o de pertenecer a una minoría que tiene dificultades para encontrar iguales (la homosexual, por ejemplo) puede estar tentadas a recurrir a los menores, porque son las personas que tiene más disponibles.

f.- Algunas personas consideran especialmente atractiva la relación emocional con los menores, sintiéndose más seguros, confortables, etc., que con personas adultas, pudiendo llegar a intimar sexualmente con ellos.

g.- Algunas personas son parafílicas, tienen una orientación de deseo sexual que solo obtiene excitación y placer sexual con prepúberes. Son las personas, varones prácticamente siempre, llamadas pedófilos. Pero éstos son una minoría en la población, de forma que la mayor parte de los abusadores sexuales no son pedófilos $y$, por otra parte, hay pedófilos responsables que autocontrolan sus deseos y no cometen abusos.

h.- Hay personas que (especialmente adolescentes) que abusan porque tienen un alto deseo sexual al que no saben encontrar salida, imitan a los adultos o lo que ven en los medios, repiten lo que hicieron con ellos otras personas, etc.

Cada una de estas causas puede actuar de manera aislada o en interacción con otras, especialmente con las señaladas, más arriba, como "culturales», que favorecen los abusos de las personas más vulnerables a cometerlos. Por ejemplo, el acceso y uso de la pornografía infantil, el apoyo de otros abusadores, etc., parece influir en personas vulnerables para que acaben cometiendo abusos sexuales.

Pero es importante señalar que sea cual sea la causa, son muy pocos los casos en los que la persona no puede evitar cometer esta conducta, por lo que hay que considerarlas siempre responsables. De hecho, la idea de que los abusadores y agresores sexuales no pueden dejar de cometer estas conductas contradice la realidad: la mayor parte de los abusadores pueden dejar de cometerlas y de hecho muchos lo hacen únicamente una vez o varias, o sólo con determinadas 
personas, o sólo en determinados momentos de su vida. Es verdad que hay una minoría, pequeña con seguridad, que parece estar sobrepasada por su falta de control y a la que no conseguimos ayudar con éxito terapéuticamente; pero en la mayoría de los caos no es así. Y, por supuesto, hay muchos que pueden ser ayudados a salir de esta situación.

\section{LA UTOPÍA DEL FUTURO: LA ÉTICA EN LAS RELACIONES AMOROSAS}

El que la sexualidad pertenezca al reino de la libertad conlleva que sea necesario plantearse los principios éticos que deben regular las relaciones sexuales, dado que pueden entrar en conflicto las libertades entre las personas.

En otro lugar hemos tratado este tema más extensamente. Baste decir aquí que consideramos básica ${ }^{13}$ :

a.- La ética de la igualdad entre las personas, de forma que, más en concreto, desaparezca cualquier forma de discriminación, también sexual, entre los hombres y las mujeres. Ambos deben tener los mismos derechos y la misma libertad para organizar su biografía sexual.

b.- La ética de consentimiento: toda actividad sexual entre dos personas debe ser consentida en condiciones de libertad de cada una de ellas. Las fronteras entre la seducción y la presión o la violencia no deben ser superadas nunca. En el caso de los menores hay que tener en cuenta que a los menores de 13 años (en España) no se le concede la capacidad de consentir tener actividad sexual con adultos, sí con iguales. Además, es preciso decir que los menores entre 13 y 18 años, especialmente en el caso de los más pequeños, en numerosas ocasiones pueden llegar consentir sin un consentimiento razonablemente informado.

c.- La ética del placer compartido, de forma que si buscar el propio placer es legítimo y deseable, no lo es menos el ayudar a la otra persona a disfrutar de su sexualidad y de la relación. Se trata de ser verdaderamente empático de forma que nos sintamos concernidos por la otra persona: tu bienestar me concierne y lo procuro, tu dolor me concierne y te consuelo y ayudo a evitarlo. La relación sexual no debe ser un encuentro entre personas ajenas que se instrumentalizan, sino entre personas que se tienen en cuenta, se saben solidarias, se apoyan y se quieren.

d.- La ética de la responsabilidad común en relación con las consecuencias, como el posible embarazo, enfermedades transmisión sexual, etc. Ambos evitan los riesgos y se apoyan y deciden evitarlos. Si el riesgo se consuma, ambos deben afrontar la situación de forma solidaria.

Sobre esta ética es posible construir relaciones en las que se dé un salto cualitativo, a partir del cual los miembros de la pareja se cuidan -es el sistema de cuidados que se ocupa del otro, sin medida, por amor-y establecen vínculos: es la utopía a la que podemos aspirar en las relaciones interpersonales.

13. LÓPEZ, Félix: "Ética de las relaciones amorosas», Acta Portuguesa de Sexología, Número especial V. Congreso nacional (2003), pp. 43-53. 